\title{
Tribological behaviors of Gra./Cu and CNTs/Cu composites with and without electric current
}

\author{
Wei $\mathrm{Xu}^{1, \mathrm{a}}$, Binghong $\mathrm{Li}^{2}$ \\ ${ }^{1}$ College of Mechanical and Electrical Engineering, Xi' an Polytechnic University, Xi' an 710048, China \\ ${ }^{2} \mathrm{Xi}$ 'an Research Institute of China Coal Technology\& Engineering Group Corp, Xi' an 710077, China
}

\begin{abstract}
Gra./Cu composites and $\mathrm{CNTs} / \mathrm{Cu}$ composites were respectively fabricated by powder metallurgy techniques. The experiments of high speed sliding with and without electric current were carried out to investigate their tribological behaviors. The results show that the friction coefficients and wear rates with electric current are higher than without electric current; under the same testing condition the friction coefficients and wear rates of CNTs/Cu are lower and the worn surfaces are more planar than $\mathrm{Gra} . / \mathrm{Cu}$. CNTs can debase the effect of heat generating during sliding process on the composites. The tribological properties of $\mathrm{CNTs} / \mathrm{Cu}$ composites are more excellent than $\mathrm{Gra} . / \mathrm{Cu}$ composites.
\end{abstract}

\section{1 introduction}

Since the discovery of carbon nanotubes (CNTs), there have been increasing interests in the field of the composites reinforced by CNTs by virtue of their physical and chemical properties such as high strength, good self-lubricating property, unique conductivity and chemical inactivity[1-3]. The nano-composites reinforced by CNTs possess extraordinary properties[4,5]. The tribological properties of CNTreinforced $\mathrm{Cu}$ matrix composites have been investigated by $\mathrm{Tu} \mathrm{JP}$ et al.[6,7]. They found that the friction coefficient of the composites is reduced and the wear resistance is enhanced due to the effect of CNTs, indicating the CNTs in the composites are not damaged during the composite preparation and play a strengthening and toughening role in the metal matrix composites. Because of its superior properties, CNTs offer tremendous opportunities for the development of fundamentally new electric contact material system.

The electric sliding wear behavior of Graphite/Cu composite material against a stainless steel band under power of maglev vehicle required was investigated by X.C.Ma et al.[8], showing that arc erosion is one of the dominant wear mechanisms during the electric sliding wear process. Gra./Cu composites have attracted much attention due to the combined properties, such as thermal and electric conductivity characteristic of copper and solid lubricating property of graphite[9-10]. In view of the wide use of $\mathrm{Gra} . / \mathrm{Cu}$ composites for electric brushes, contact strips and bearing materials in many application[11], it is supposed that $\mathrm{Cu}$ matrix composites reinforced by CNTs, instead of graphite, would have higher mechanical strength, thermal conductivities, arc extinguishing properties, and wear resistivity, which is scarcely reported so far according to our knowledge. So, in this paper we comparatively investigate the friction and wear behavior of $\mathrm{Gra} . / \mathrm{Cu}$ and $\mathrm{CNTs} / \mathrm{Cu}$ composites with and without electric current.

\section{Experiment}

\subsection{Preparation of composites}

The outer diameter of CNTs is less than 20nm, the length $10 \sim 30 \mu \mathrm{m}$ and the purity $95 \%$. The initial CNTs showed in twist and cluster[12]. So, they were mechanically milled for $5 \mathrm{~h}$ in an organic liquid by a planetary ball mill machine, which should make them into shorter pieces and more symmetrical dispersion in the matrix. CNTs were purified by being immersed in the mixture of nitric acid and sulphuric acid, then filtered and washed with de-ionized water and dried at $120^{\circ} \mathrm{C}$.

The composites were fabricated by the powder metallurgy technique and the process included mixing powder, compacting and sintering in vacuum atmosphere. The microstructure images of $\mathrm{Cu}$ matrix composites are respectively shown in Fig.1, and it can be found that CNTs and graphite appear dispersive and are fully embedded in the copper matrix. The physical characteristics are shown in Tab.1, and the physical characteristics of $\mathrm{CNTs} / \mathrm{Cu}$ composite are better than those of Gra./Cu composite.

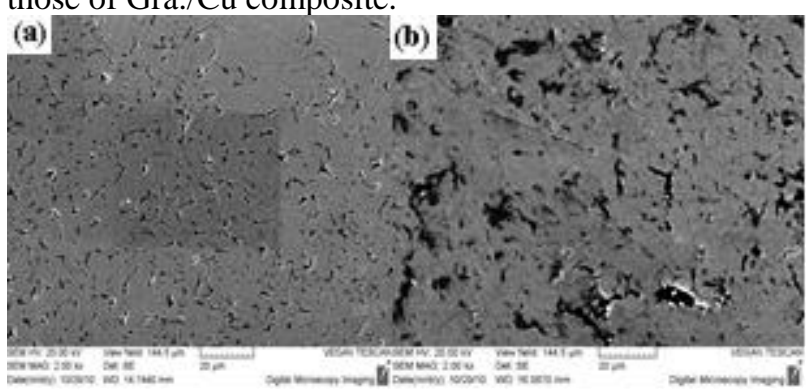

Fig.1 SEM image of copper matrix composite 
(a) $\mathrm{CNTs} / \mathrm{Cu}$; (b) Gra./Cu.

Tab.1 Physical characteristics of the Gra./Cu and CNTs/Cu composites

\begin{tabular}{ccccc}
\hline & $\begin{array}{c}\text { Density } \\
(\mathrm{g} / \mathrm{cm} 3)\end{array}$ & $\begin{array}{c}\text { Hardness } \\
(\mathrm{HB})\end{array}$ & $\begin{array}{c}\text { Compactness } \\
(\%)\end{array}$ & $\begin{array}{c}\text { Thermal } \\
\text { conductivity } \\
(\mathrm{W} /(\mathrm{m} . \mathrm{k}))\end{array}$ \\
$\mathrm{Gra} . / \mathrm{Cu}$ & 7.5 & 53 & 97 & 248.267 \\
$\mathrm{CNTs} / \mathrm{Cu}$ & 7.4 & 55 & 97 & 326.026 \\
\hline
\end{tabular}

\subsection{Tests measurements}

Those experiments of high speed sliding with 40A electric current and without electric current were carried out at a sliding speed of $15 \mathrm{~m} / \mathrm{s}$ and at an applied load of $20 \mathrm{~N}$ to investigate the friction and wear behaviors of the $\mathrm{Cu}$ matrix composites by using a pin-on-disk friction and wear tester. All specimens were polished with 1000 mesh $\mathrm{SiC}$ paper and degreased with acetone before every experiment. The counterpart used as the disk in the experiments had a diameter of $800 \mathrm{~mm}$ and was fabricated from the $\mathrm{Cu}-0.5 \mathrm{Cr}$ alloy. The friction coefficients were calculated by dividing the friction force which was recorded on line via torque as measured by the strain gauge, by the applied load. The wear resistance was evaluated according to the weight loss of the specimen after testing, and the weight loss was measured with an analytical balance. In order to take repeatability into account, the test results of wear rate under steady sliding were obtained from the average of three readings. Scanning electron microscope was used to observe the worn surfaces of the composites, which would not be cleaned in order to observe all the features including the wear scars and the surface films on the worn surface.

\section{Rults and Discussion}

\subsection{Friction coefficient of the composites}

The friction coefficients of the $\mathrm{Cu}$ matrix composites under diverse experiment conditions are presented in Fig.2, and the average values of the friction coefficients are respectively shown in Tab.2. It can be noticed that the friction coefficients of CNTs/Cu composite are lower than $\mathrm{Gra} . / \mathrm{Cu}$ composite under the same test condition. It is suggested that the short and tube shape of CNTs would more easily slide or roll between the contact surfaces, thus hindering the deformation of $\mathrm{Cu}$ matrix during the sliding process, and resulting in the decrease of friction coefficients. On the other hand, it is thought that the high temperature is a key influence factor for friction and wear behavior of composites[13]. CNTs/Cu composites possess more prominent thermal conductivity than $\mathrm{Gra} / \mathrm{Cu}$ composites, which can decrease the effect of friction heat and Joule heat on the lubricating film of the composites, and ultimately enhance the anti-friction ability of $\mathrm{Cu}$ matrix composites.
Tab.2 The average values of friction coefficients of the $\mathrm{Cu}$ matrix composites under diverse experiment

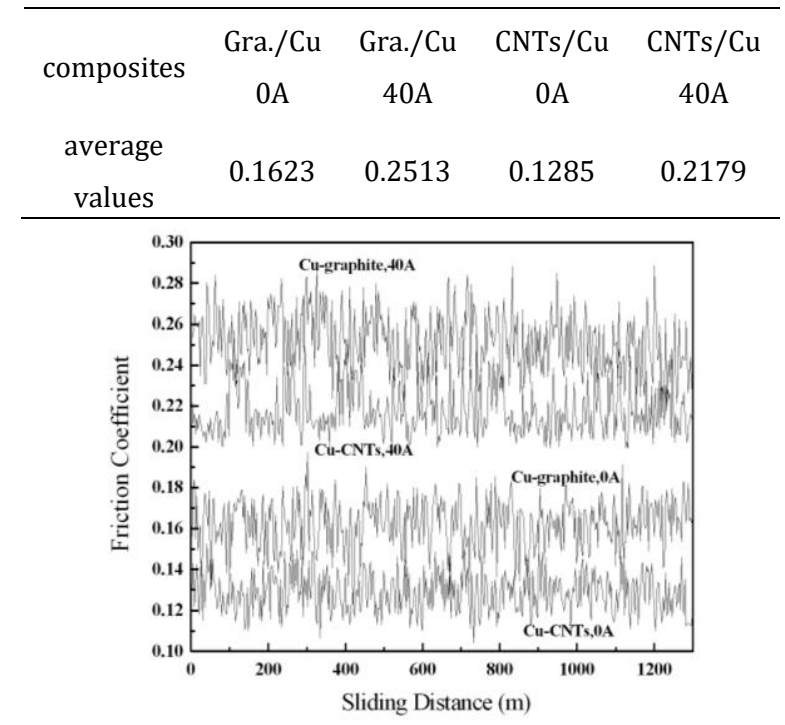

Fig.2 Friction coefficients of the $\mathrm{Cu}$ matrix composites under diverse experiment conditions

From Fig.2 and Tab.2, it can also be noted that the friction coefficients of $\mathrm{Cu}$ matrix composites with $40 \mathrm{~A}$ electric current are higher than without electric current. In electric wear process the total power loss is the sum of mechanical loss and electric loss[14,15], and the combined effects of mechanical loss and electric loss lead to extremely high local temperature. Under the condition of high temperature, the continuity and integrity of lubricating film on the surface of composites are damaged, and finally the roughness of worn surface is increased, which can explain why the friction coefficient is higher during the sliding process with electric current.

\subsection{War rate of the composites}

Fig. 3 shows the wear rates of the $\mathrm{Cu}$ matrix composites under diverse experiment conditions. As can be seen, the wear rates of $\mathrm{CNTs} / \mathrm{Cu}$ composites are lower than Gra./Cu composites under the same test condition, which mainly owes to the action of reinforcement and lubrication of CNTs. Both CNTs and graphite have excellent lubrication characteristic, their debris gradually spread out of the contact interface forming a layer of lubricating film, however CNTs have higher strength than graphite to resist cutting of the counterpart, and can enhance the microhardness of $\mathrm{Cu}$ matrix composites obviously, which leads to the decrease of the wear rate. So CNTs play a strengthening and toughening role in the metal matrix composites[16]. In addition, CNTs possess remarkable electric and thermal conductivity which decreases the effect of electric arc and heat on wear resistance of the composites.

From Fig.3, it can also be seen that the wear rate of the composites with $40 \mathrm{~A}$ electric current is higher than those without electric current. With electric current, the total amount of heat produced during wear process comes from three symptoms: electric arc heat, friction 
heat and Joule heat[15]. The temperature of the arc plasma at the electrode is known to reach up to 3500$4000 \mathrm{~K}[8,17]$. The temperature of the surface and the subsurface where the arc is discharged increases promptly, which makes the composites be intenerated and deformed by friction force at high temperature and ultimately results in the increase of wear rate.

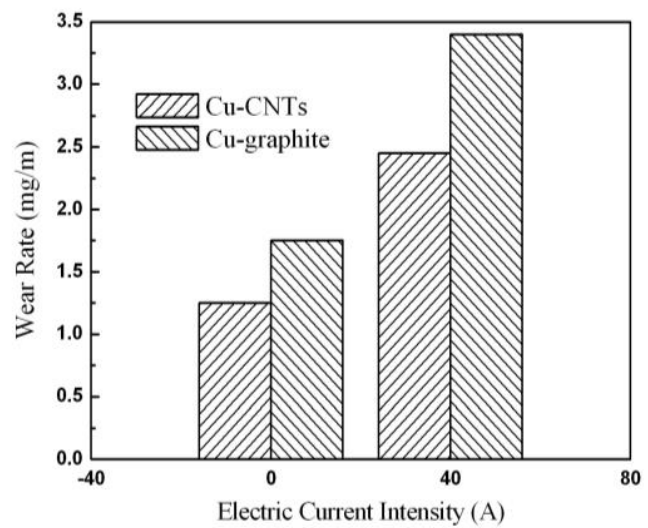

Fig.3 Wear rates of $\mathrm{Cu}$ matrix composite under diverse experiment conditions

\subsection{Worn micrographs of the composites}

Fig.4 shows the worn surface SEM micrograph of Gra./Cu composites. As can be observed, the worn surfaces possess disparate morphology, which indicates different wear process. As shown in Fig.4(a), there are a number of deep grooves and cracks on the worn surface, indicating that abrasive and adhesive wear have happened in the testing without electric current. During the sliding process, accumulation of plastic deformation induced by shearing strength can cause numerous microcracks, which would grow and extend to the surface. Then many pieces of the split copper would form on the worn surface. As the Cu-matrix gradually flakes, graphite on the surface and subsurface of the composite would be exposed and become the working film, and the worn contacting surfaces are thus changed from the original metal surfaces into metals with a lubricating carbon film. As plasticity of the film is sensitive to temperature, the friction heat causes an increase in local temperature. When the temperature is over $500{ }^{\circ} \mathrm{C}$, plasticity of the lubricating film reduces rapidly, and the flake breaks off.

There is much curved flake, debris and melted dripping besides groove and crack on the worn surface as shown in Fig.4(b). The worn surface with electric current is destroyed more severely than without electric current. In the sliding wear with electrical current process, electric arc which can release electric arc heat and Joule heat on the contact spots is induced between the cycling of contact and separation of tribology pairs. The low melting point metal-copper is partially introduced into thermal melting, as the temperature of the composite surface and subsurface sharply increases under the electric arc heat. Then the semi-molten surface cools rapidly, causing the particles to adhere to the surface in the flaking area. The combination of thermal and mechanical shock creates condition for the development of rupture and failure of surface layer and leads to intensification of wear of the composite. The Gra./Cu composite can melt locally resulting in severe arc erosion. Electric arc erosion wear and adhesive wear are the main wear mechanisms for $\mathrm{Gra} . / \mathrm{Cu}$ composite during the sliding wear with $40 \mathrm{~A}$ electric current.

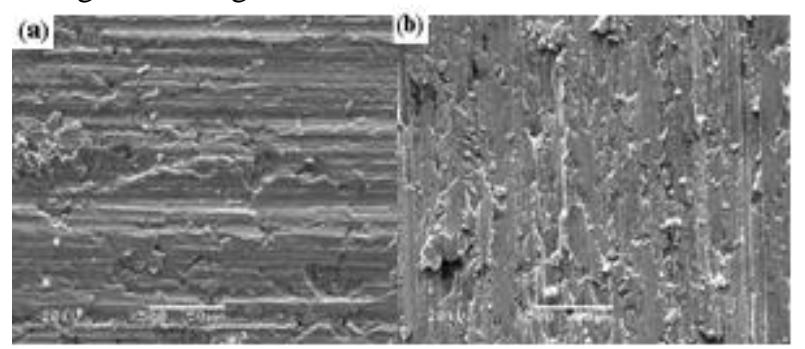

Fig.4 The worn micrograph of Gra./Cu composite

(a) without electric current; (b) with 40A electric current

Fig.5(a) shows the worn surface SEM micrograph of $\mathrm{CNTs} / \mathrm{Cu}$ composite without electric current. It can be seen that there are flake-like wear scars on the worn surface, which appears to be a typical characteristic of adhesive wear and more planar than Gra./Cu composite under the same testing condition. CNTs possess not only the lubricating characteristic of graphite but also higher strength and more excellent thermal conductivity than graphite. Thus CNTs instead of graphite can decrease the effect of friction force and heat generated from the sliding wear on the $\mathrm{Cu}$ matrix composites. The lubricating film consist of CNTs can reduce the wear surface exposed in the air, and prevent the $\mathrm{Cu}$ matrix from oxidizing. So the adhesive between the composite and the counterpart can be significantly reduced. In brief, the $\mathrm{Cu}$ matrix composites reinforced by CNTs are provided with more outstanding anti-friction and wear resistance than those reinforced by graphite under the condition of sliding wear.

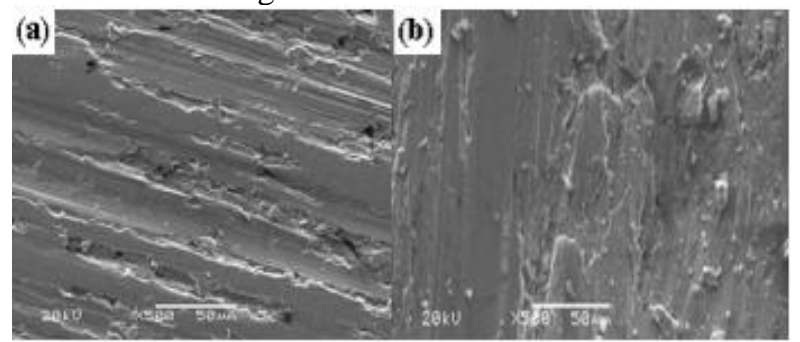

Fig.5 The worn micrograph of CNTs/Cu composite (a) without electric current; (b) with 40A electric current

The worn surface SEM micrograph of CNTs/Cu composites with 40A electric current is shown in Fig.5(b). Plastic deformation with characteristic wear scars and a low-degree flake formation, which are produced by crashing and shearing under the friction force around the contact area during the process of wear, can be observed on the worn surface of $\mathrm{CNTs} / \mathrm{Cu}$ composite. Additionally, on the worn surface some scraps and cracking of the flake layer but no remarkable melted dripping can be found. From Fig.5(a) and (b), the worn surface is destroyed more severely in the occasion of sliding wear with electric current. Electric arc causes more quantity of heat resulting in an increase of temperature which inhibits the ability of the film to remain tightly bound to the matrix material. The film 
layer become flaky, discontinuous and easily removed so that further wear would take place.

\section{Conclusions}

This paper mainly focuses on the friction and wear behaviors of $\mathrm{Gra} . / \mathrm{Cu}$ and $\mathrm{CNTs} / \mathrm{Cu}$ composites with and without electric current. The following conclusions can be drawn from this study.

(1) Temperature is a key influence factor for friction and wear behavior of the $\mathrm{Cu}$ matrix composites. Electric current cause more quantity of heat resulting in an increase of temperature. The friction coefficients and wear rates of the $\mathrm{Cu}$ matrix composites with electric current are higher than those without electric current.

(2) The friction coefficients and wear rates of $\mathrm{CNTs} / \mathrm{Cu}$ composite are lower than those of $\mathrm{Gra} . / \mathrm{Cu}$ composite under the same test condition. The tubular CNTs possess not only excellent lubricating characteristic but also high strength and remarkable electric and thermal conductivity, which ultimately induce the decrease of friction coefficients and wear rates of the $\mathrm{Cu}$ matrix composites.

(3) The worn surfaces of the composites with 40A electric current are destroyed more severely than those without electric current. The worn surfaces of $\mathrm{CNTs} / \mathrm{Cu}$ composite appear to be more planar than those of $\mathrm{Gra} / \mathrm{Cu}$ composite under the same test condition. The $\mathrm{Cu}$ matrix composites reinforced by $\mathrm{CNTs}$ are provided with more outstanding anti-friction and wear resistance than those reinforced by graphite under the condition of sliding wear with and without electric current.

\section{Acknowledgements}

Foundation item: Xi'an Polytechnic University Research Fund for the Doctoral Program(BS1303);

\section{References}

1. Q.M. Gong, D Li, Z Li. Tribology and Interface Engineering Series. 55, 245(2008)

2. J.M. Zhang, T Masataka, M.Toshiro. Computer Methods in Applied Mechanics and Engineering. 193,559 (2004)

3. W.T.Hong, N.H.Tai. Diamond and Related Materials, , 17,1577( 2008)

4. W.X.Chen, J.P. Tu, L.Y.Wang. Carbon. 41, 215(2003)

5. S.M.Zhou, X.B. Zhang, Z.P. Ding. Composites Part A, 38, 301(2007)

6. S.R.Dong, J.P.Tu, X.B.Zhang. Material science and engineering, 313 ,83(2001)

7. J.P.Tu, Y.Z.Yang, L.Y.Wang. Tribology Letters, 10,225(2001)

8. X.C.Ma, G.Q. He, D.H.He. Wear, 265, 1087(2008)

9. $\mathrm{R} \mathrm{R}$ Zahran, I HM Ibrahim, GH Sedahmed. Materials Letters, 28,237(1996)
10. S F Moustafa, S A Ei Badry, AM Sanad, Wear, 253, 699(2002)

11. H.J.Zhao,L. Liu, Y.T.Wu. Composites Science and Technology, 67,1210( 2007)

12. S Ijima. Nature, $\mathbf{3 5 4 , 5 6 ( 1 9 9 1 )}$

13. H Zhao, G.C. Barber,J. Liu. Wear, 249,409(2001)

14. J Wang, Y Feng, S Li. Transactions of Nonferrous Metals Society of China, 19, 113(2009)

15. Y Feng, M Zhang, Y Xu. Carbon, 43, 2685(2005)

16. S.M. Zhou, X.B.Zhang, Z.P.Ding. Composites Part A, 38, 301(2007)

17. S.G.Jia, P.Liu, F.Z.Ren. Materials Science and Engineering A, 398,262(2005) 